\title{
Measurement of the forward-backward asymmetry in top production at CDF
}

\author{
Costas Vellidis*广 \\ FNAL \\ E-mail: vellidisefnal.gov
}

This article reports measurements of the forward-backward asymmetry in top quark pair production with $p \bar{p}$ collisions at a total energy $\sqrt{s}=1.96 \mathrm{TeV}$. The asymmetry is measured in both the lepton + jets $(l j)$ and the dilepton $(l l)$ decay channels using data corresponding to integrated luminosities of $5.3 \mathrm{fb}^{-1}$ and $5.1 \mathrm{fb}^{-1}$, respectively, recorded with the CDF detector. The inclusive hadron-level asymmetries in the $p \bar{p}$ rest frame (laboratory), fully corrected for background contamination, detector acceptance and finite resolution effects, are found to be consistent with $\mathrm{CP}$ conservation under interchange of top and anti-top quarks and they are $A_{\text {exp }}^{l j}(p \bar{p})=0.158 \pm 0.072_{\text {stat }} \pm 0.017_{\text {syst }}$ in the $l j$ channel and $A_{\text {exp }}^{l l}(p \bar{p})=0.42 \pm 0.15_{\text {stat }} \pm 0.05_{\text {syst }}$ in the $l l$ channel. These results are to be compared with next-to-leading order (NLO) QCD predictions of $A_{t h}^{l j}(p \bar{p})=0.018 \pm 0.005$ and $A_{t h}^{l l}(p \bar{p})=0.06 \pm 0.01$, respectively. The measurements in the two channels are combined to give $A_{\text {exp }}^{l j+l l}(p \bar{p})=0.20 \pm 0.07_{\text {stat }} \pm 0.02_{\text {syst }}$. In the $l j$ channel, parton-level asymmetries are derived in two regions of the $t-\bar{t}$ rapidity difference $\Delta y$ and two regions of the invariant mass $M_{t \bar{t}}$. The asymmetry is found most significant at large $\Delta y$ and $M_{t \bar{t}}$, with a result in the $t \bar{t}$ rest frame of $A_{\text {exp }}^{l j}(t \bar{t})=0.475 \pm 0.114_{t o t}$ compared to a NLO QCD prediction of $A_{t h}^{l j}(t \bar{t})=0.088 \pm 0.013$.

XXIst International Europhysics Conference on High Energy Physics

21-27 July 2011

Grenoble, Rhône-Alpes France

\footnotetext{
${ }^{*}$ Speaker.

${ }^{\dagger}$ On behalf of the CDF Collaboration.
} 
Leading-order (LO) QCD does not discriminate between quark and anti-quark in heavy flavor pair production via $q \bar{q}$ annihilation or gg fusion. In NLO QCD, however, radiative corrections involving a real or a virtual gluon in $q \bar{q} \rightarrow Q \bar{Q}$ lead to a difference in the production of $Q$ and $\bar{Q}$ and consequently, assuming $\mathrm{CP}$ conservation, to a small but non-zero charge asymmetry originating from the interference of charge even and odd diagrams. The overall charge asymmetry is positive and of the order of a few percent [1]. New physics can also generate an asymmetry, through mechanisms involving axigluons, diquarks, new weak bosons, and extra dimensions [2], without significantly modifying the $t \bar{t}$ production cross section and $M_{t \bar{t}}$ distribution at Tevatron energies, which have been measured to be consistent with standard model (SM) predictions [3].

In the $l j$ channel [4], the asymmetry is measured from a sample of $t \bar{t}$ event candidates using the event selection, event reconstruction and background estimation algorithms applied in the top quark mass [5] and lepton+jets cross section [3] measurements. Assuming CP symmetry, the rapidity of the hadronic top is used to measure the asymmetry in the $p \bar{p}$ rest frame (laboratory) and the $t-\bar{t}$ rapidity difference is used to measure the asymmetry in the $t \bar{t}$ rest frame. The inclusive asymmetry in either frame is corrected for background contributions and for the detector finite acceptance and resolution, with or without correction for parton showering, to derive a partonlevel or a hadron-level asymmetry, respectively. Alternatively, the asymmetry is binned over the $t-\bar{t}$ rapidity difference $\Delta y$ or the invariant mass $M_{t \bar{t}}$ and the corrections are applied on the binned asymmetry in either frame. The variables $\Delta y$ and $M_{t \bar{t}}$ are kinematically correlated and thus the asymmetry has similar dependence on both. Systematic uncertainties in the corrected asymmetry are estimated for the background size and shape, physics model, jet energy scale, gluon radiation, color reconnection and proton PDF, and they are added in quadrature to determine the overall systematic uncertainty. The dominant source of systematic uncertainty is the physics model.

In the $l l$ channel [6], the asymmetry is measured from a sample of $t \bar{t}$ event candidates using the event selection and background estimation applied in the dilepton cross section measurement for $t \bar{t}$ production [7], with the additional selection requirement of the two leptons coming from the same vertex. An algorithm for the reconstruction of both the $t$ and $\bar{t}$ directions is developed which depends on the topology of $t \bar{t}$ events. Corrections are applied to the raw asymmetry of the $t-\bar{t}$ rapidity difference for background contributions and for the detector finite acceptance and resolution in order to derive a hadron-level asymmetry comparable to theoretical predictions. Systematic uncertainties in the corrected asymmetry are estimated for the background shape, detector modeling, signal modeling, jet energy scale, gluon radiation, color reconnection and proton PDF, and they are added in quadrature to determine the overall systematic uncertainty. The dominant source of systematic uncertainty is the background shape.

Since the $l j$ and $l l$ samples are mutually exclusive, the asymmetry measurements from the two samples can be combined into one with reduced statistical uncertainty [8]. The combination is done by computing the effective forward and backward events from both results, adding them individually and then computing the final asymmetry from the total numbers of effective forward and backward events. Systematic uncertainties are propagated by fluctuating the central asymmetry value of each measurement by one standard deviation up and down for each source of uncertainty, recomputing the numbers of effective forward and backward events and deriving the fluctuated asymmetry. The average deviation from the central combined value is quoted as the systematic uncertainty from each source which is then added in quadrature to the total systematic uncertainty 


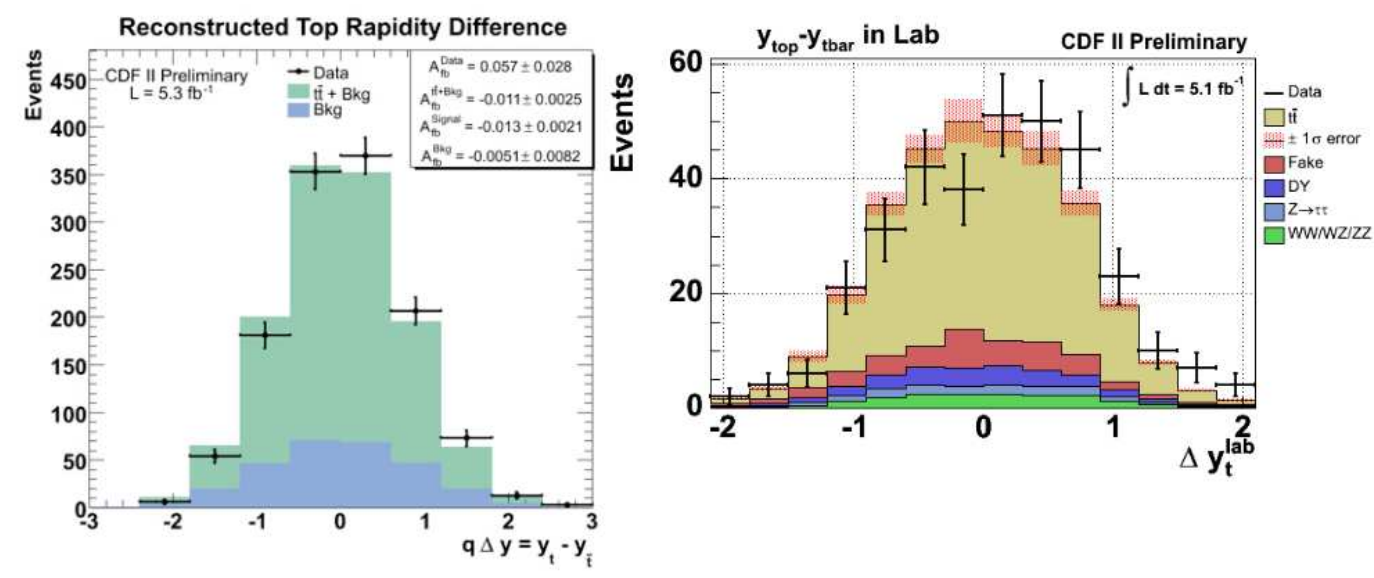

Figure 1: The measured $t-\bar{t}$ rapidity difference in the $l j$ (left) and $l l$ (right) channels compared with LO QCD predictions.

of the final result.

Figure 1 shows the $t-\bar{t}$ rapidity difference distribution measured in each decay channel and compared with LO QCD predictions for the signal and the background. A positive asymmetry, consistent with CP conservation, is observed in both channels. The hadron-level corrected asymmetries in the laboratory frame are $A_{\text {exp }}^{l j}(p \bar{p})=0.158 \pm 0.072_{\text {stat }} \pm 0.017_{\text {syst }}$ and $A_{\text {exp }}^{l l}(p \bar{p})=0.42 \pm$ $0.15_{\text {stat }} \pm 0.05_{\text {syst }}$, both likely stronger than, although not entirely inconsistent with, the respective NLO QCD predictions for the signal asymmetry of $A_{t h}^{l j}(p \bar{p})=0.018 \pm 0.005$ and $A_{t h}^{l l}(p \bar{p})=$ $0.06 \pm 0.01$. Theoretical uncertainties include Monte Carlo statistics and scale uncertainties. The combination of the two measurements gives $A_{\text {exp }}^{l j+l l}(p \bar{p})=0.20 \pm 0.07_{\text {stat }} \pm 0.02_{\text {syst }}$, a result of 2.9 standard deviations away from the symmetric hypothesis. Figure 2 shows on the left the $t-\bar{t}$ rapidity difference distribution in the $l j$ channel in two bins of low (top, $M_{t \bar{t}}<450 \mathrm{GeV} / \mathrm{c}^{2}$ ) and high (bottom, $M_{t \bar{t}}>450 \mathrm{GeV} / \mathrm{c}^{2}$ ) $t \bar{t}$ invariant mass. While in the low mass bin the asymmetry is consistent with zero, in the high mass bin it is clearly positive and strong. The parton-level corrected asymmetry is $A_{\text {exp }}^{l j}\left(M_{t \bar{t}}<450\right)=-0.116 \pm 0.146_{\text {stat }} \pm 0.047_{\text {syst }}$ and $A_{\text {exp }}^{l j}\left(M_{t \bar{t}}>450\right)=$ $0.475 \pm 0.101_{\text {stat }} \pm 0.049_{\text {syst }}$, and it is compared in the top right window with a NLO QCD prediction of $A_{t h}^{l j}\left(M_{t \bar{t}}<450\right)=0.040 \pm 0.006$ and $A_{t h}^{l j}\left(M_{t \bar{t}}>450\right)=0.088 \pm 0.013$. The measurement is 3.4 standard deviations away from the prediction in the high mass bin.

In summary, the forward-backward asymmetry in $t \bar{t}$ production is measured in both the $l j$ and $l l$ channels and the results are combined. These measurements provide a test of charge asymmetry in the strong interaction at large momentum transfer. All results show a positive asymmetry, consistent with CP conservation, at the level of 2-3 standard deviations larger than NLO QCD predictions. In the $l j$ channel, in particular, the study of the kinematic dependences of the asymmetry indicates a strong dependence on the $t \bar{t}$ invariant mass.

\section{References}

[1] L. G. Almeida, G. F. Sterman and W. Vogelsang, Phys. Rev. D 78, 014008 (2008); O. Antunano, J. H. Kuhn, and G. V. Rodrigo, Phys. Rev. D 77, 014003 (2008); M. T. Bowen, S. D. Ellis, and D. 

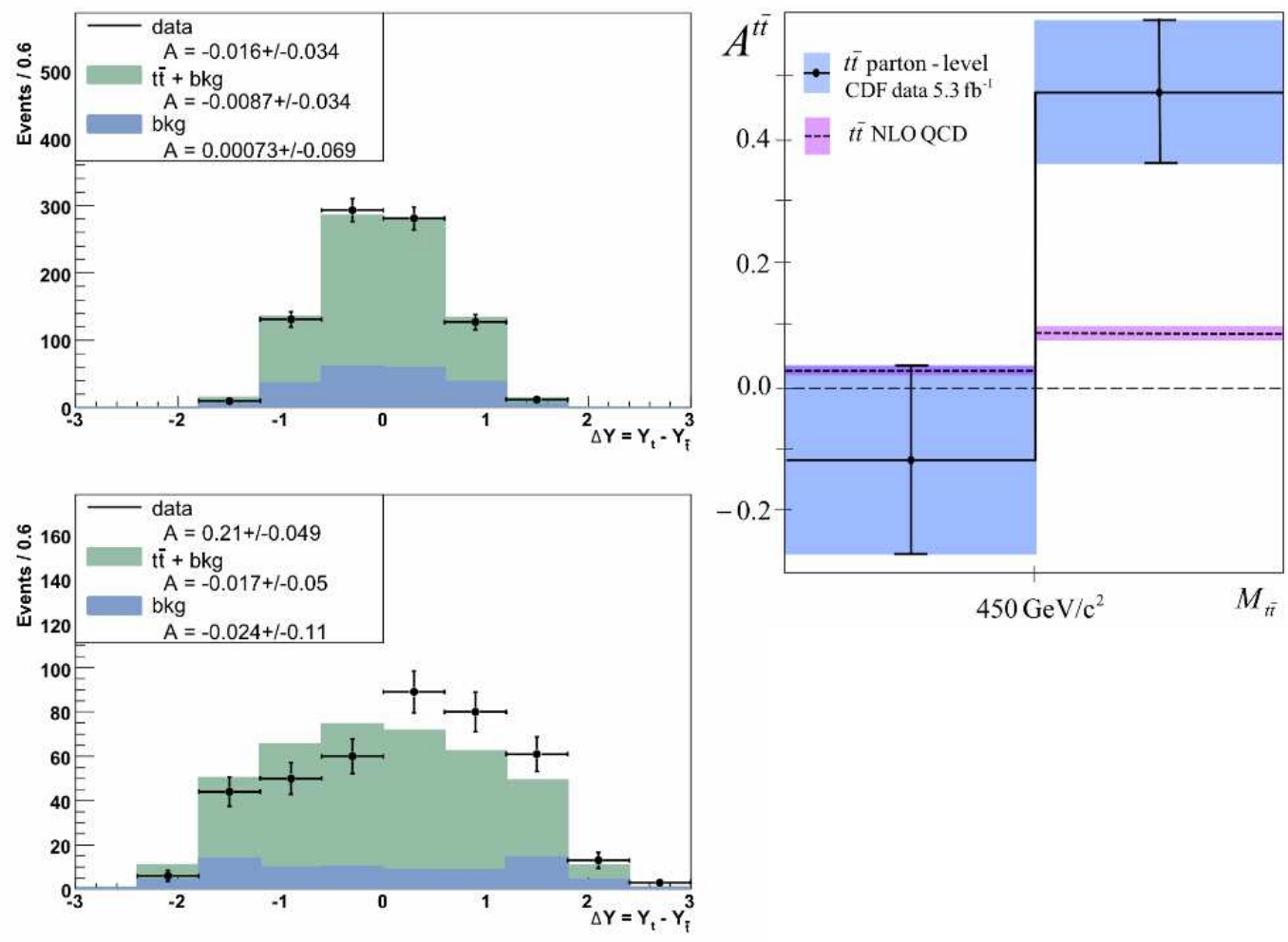

Figure 2: Left: The measured $t-\bar{t}$ rapidity difference in the $l j$ channel in two bins of low (top left) and high (bottom left) invariant mass $M_{t \bar{t}}$ compared with LO QCD predictions. Right: The parton-level asymmetry as a function of $M_{t \bar{t}}$ compared with a NLO QCD prediction.

Rainwater, Phys. Rev. D 73, 014008 (2006); S. Moch and P. Uvwer, Nucl. Phys. Proc. Suppl. 183, 75 (2008).

[2] D. W. Jung, P. Ko, J. S. Lee, and S. H. Nam, Phys. Lett. B 691, 238 (2010); M. Bauer, F. Goertz, U. Haisch, T. Pfoh, and S. Westhoff, J. High Energy Phys. 11 (2010) 039; R. S. Chivukula, E. H. Simmons, and C. P. Yuan, Phys. Rev. D D 82, 094009 (2010); B. Xiao, Y. K. Wang, and S. H. Zhu, Phys. Rev. D D 82, 034026 (2011); Q.-H. Cao, D. McKeen, J. Rosner, G. Shaughnessy, and C. Wagner, Phys. Rev. D D 81, 114004 (2010); I. Dorsner, S. Fajfer, J. F. Kamenik, and N. Kosnik, Phys. Rev. D D 81, 055009 (2010); S. H. Jung, H. Muryama, A. Pierce, and J D. Wells, Phys. Rev. D D 81, 015004 (2010); P. H. Frampton, J. Shu, and K. Wang, Phys. Lett. B 683, 294 (2010); J. Shu, T. M. P. Tait, and K. Wang, Phys. Rev. D D 81, 034012 (2010); V. Barger, W.-Y. Keung, and C.-T. Yu, Phys. Rev. D D 81, 113009 (2010).

[3] T. Aaltonen et al. (CDF Collaboration), Phys. Rev. Lett. 105, 012001 (2010); T. Aaltonen et al. (CDF Collaboration), Phys. Rev. Lett. 102, 222003 (2009).

[4] T. Aaltonen et al. (CDF Collaboration), Phys. Rev. D 83, 112003 (2011).

[5] A. Abulencia et al. (CDF Collaboration), Phys. Rev. D 73, 032003 (2006).

[6] T. Aaltonen et al. (CDF Collaboration), CDF Public Note 10436 (2011).

[7] T. Aaltonen et al. (CDF Collaboration), CDF Public Note 10163 (2010).

[8] T. Aaltonen et al. (CDF Collaboration), CDF Public Note 10584 (2011). 